\title{
Factors Affecting the Motor Evoked Potential Responsiveness and Parameters in Patients With Supratentorial Stroke
}

\author{
Tae Woong Choi, MD, Seung Gul Jang, MD, \\ Seung Nam Yang, MD, PhD, Sung-Bom Pyun, MD, PhD \\ Department of Physical Medicine and Rehabilitation, Korea University Hospital, \\ Korea University College of Medicine, Seoul, Korea
}

\begin{abstract}
Objective To investigate the factors which affect the motor evoked potential (MEP) responsiveness and parameters and to find the correlation between the function of the upper extremities and the combined study of MEP with a diffusion tensor tractography (DTT) in patients with stroke.

Methods A retrospective study design was used by analyzing medical records and neuroimaging data of 70 stroke patients who underwent a MEP test between June 2011 and March 2013. MEP parameters which were recorded from the abductor pollicis brevis muscle were the resting motor threshold, latency, amplitude, and their ratios. Functional variables, Brunnstrom stage of hand, upper extremity subscore of Fugl-Meyer assessment, Manual Function Test, and the Korean version of Modified Barthel Index (K-MBI) were collected together with the biographical and neurological data. The DTT parameters were fiber number, fractional anisotropy value and their ratios of affected corticospinal tract. The data were compared between two groups, built up according to the presence (MEP-P) or absence (MEP-N) of MEP on the affected hand.

Results Functional and DTT variables were significantly different between MEP-P and MEP-N groups $(\mathrm{p}<0.001)$. Among the MEP-P group, the amplitude ratio (unaffected/affected) was significantly correlated with the Brunnstrom stage of hand $(\mathrm{r}=-0.427, \mathrm{p}=0.013), \mathrm{K}-\mathrm{MBI}(\mathrm{r}=-0.380, \mathrm{p}=0.029)$ and the time post-onset $(\mathrm{r}=-0.401$, $\mathrm{p}=0.021$ ). The functional scores were significantly better when both MEP response and DTT were present and decreased if one or both of the two studies were absent.

Conclusion This study indicates MEP responsiveness and amplitude ratio are significantly associated with the upper extremity function and the activities of daily living performance, and the combined study of MEP and DTT provides useful information.
\end{abstract}

Keywords Motor evoked potential, Diffusion tensor imaging, Stroke, Motor function, Activities of daily living

Received May 16, 2013; Accepted September 24, 2013

Corresponding author: Sung-Bom Pyun

Department of Physical Medicine and Rehabilitation, Korea University Anam Hospital, Korea University College of Medicine, 73 Inchon-ro, Seongbukgu, Seoul 136-705, Korea

Tel: +82-2-920-6480, Fax: +82-2-929-9951, E-mail: rmpyun@korea.ac.kr

() This is an open-access article distributed under the terms of the Creative Commons Attribution Non-Commercial License (http://creativecommons.org/ licenses/by-nc/3.0) which permits unrestricted noncommercial use, distribution, and reproduction in any medium, provided the original work is properly cited. Copyright $\odot 2014$ by Korean Academy of Rehabilitation Medicine 


\section{INTRODUCTION}

Stroke is a leading cause of serious long-term disabilities like hemiparesis, cognitive impairment, and speech disorder. Among those, hemiparesis is the most frequent and the most disabling problem which leads to an incomplete recovery in the most of cases [1]. Therefore, an accurate measurement of motor functions is very important to provide useful information about the current status and for the optimization of rehabilitation interventions. Many studies were already conducted for an outcome prediction of motor weakness using various objective tools, including radiologic [2], electrophysiological $[3,4]$, and functional neuroimaging methods $[5,6]$.

The motor evoked potential (MEP) is the standard measure of motor response to the transcranial magnetic stimulation (TMS). This test provides in a safe and painless way information about the physiologic integrity of the corticospinal pathway from the primary motor cortex to the spinal motor neurons and also to the electrical potential in individual muscles [7]. In many previous studies where MEP was used a better functional recovery prognosis was shown for those patients with a present MEP than for those patients with an absent MEP [4,810]. Some studies have also shown the amplitude of MEP could be an effective parameter $[11,12]$. However, in other studies the inconsistency of amplitude value which varies across individuals and environments was emphasized $[13,14]$. Therefore, the use of an amplitude ratio by comparing MEP amplitudes obtained from both hemispheres may be more helpful. Also the amplitude ratio showed a significant correlation with the hand function in a previous study [15]. However, there is only a small number of studies yet where the role of amplitude ratio in the motor function assessment was highlighted. So further studies are needed to confirm those suggested correlations.

The diffusion tensor tractography (DTT), derived from the diffusion tensor imaging (DTI), is a newly developed magnetic resonance imaging (MRI) technique. DTT provides the visualization and evaluation of the corticospinal tract (CST) orientation and integrity [1618]. The predictive value of DTT for the motor outcome in stroke patients was proved in several studies [19-22]. The combined tests of TMS and DTT also showed their usefulness in the prediction of motor outcome in stroke patients $[23,24]$. However, further studies involving more parameters for MEP and DTT are needed to increase the predictability for motor and functional outcomes.

In the current study, the primary purpose was to investigate the factors affecting the MEP responsiveness and parameters. The secondary purpose was to find the correlation between upper the extremity function and the combination of MEP with DTT.

\section{MATERIALS AND METHODS}

\section{Subjects}

A retrospective study design was used by analyzing the medical records of patients who had cerebrovascular disorders and received MEP tests in our clinic between June 2011 and March 2013. Among 121 patients, a total of 70 patients with 35 males and 35 females and a mean age of $62.59 \pm 12.38$ years was included according to the following criteria: 1) first stroke ever, 2) unilateral hemispheric lesion, 3) supratentorial lesion, and 4) within 3 months after stroke onset. Patients with a previous history of stroke, bilateral hemispheric or infratentorial lesion and subarachnoid hemorrhage were excluded. The study protocol was approved by the Institutional Review Board of the university hospital.

\section{Motor evoked potential}

Patients were seated on a comfortable chair and the surface electromyography of the abductor pollicis brevis (APB) muscle was recorded. TMS was applied through a figure-of-eight coil (model C-B60) using MagPro X100 magnetic stimulators (Medtronic, Skovlunde, Denmark). MEPs were obtained from both APB muscles in a resting state. The resting motor threshold (RMT) was defined as the minimum stimulus intensity to obtain an MEP response greater than $50 \mu \mathrm{V}$ in 5 of 10 trials. Three stimulations were performed each time with intensity set at $120 \%$ of RMT, and the MEP with mid-latency and mid-amplitude was adopted. MEP tests were performed within 5 days after admission to the rehabilitation department in most of the patients. The mean duration time from stroke onset until the test date was $29.75 \pm 14.64$ days (range, 7-84 days). The patients were subdivided according to the presence or absence of MEP response on the affected APB (MEP-P group vs. MEP-N group). The ratio of latency and amplitude was calculated in MEP-P group, using a formula dividing unaffected value by affected value. 


\section{Diffusion tensor tractography}

DTI were acquired using a 3.0-T Philips Gyroscan Intera MRI scanner (Philips Healthcare, Best, The Netherlands) with a six-channel phased array sensitivity encoding (SENSE) head coil. Images were obtained based on the single-shot echo-planar image sequence. The DTI data parameters were as follows: field of view $224 \times 224 \mathrm{~mm}^{2}$; matrix $112 \times 112$; repetition time $(\mathrm{TR})=9,131 \mathrm{~ms}$; echo time (TE) $=85 \mathrm{~ms}$; slice thickness $=2 \mathrm{~mm}$; SENSE factor $=2$; and $\mathrm{b}=1000 \mathrm{~s} / \mathrm{mm}^{2}$. We obtained $70-75$ gapless, contiguous transverse images with applying along each of 32 noncollinear diffusion-sensitizing gradients.

Fiber tracking was analyzed using the "FiberTrak" package included in the Extended MR Workspace $\mathrm{R}$ 2.6.3.1 software (Philips Healthcare). The termination criteria used were fractional anisotropy (FA) $<0.2$ and an angle change $>30^{\circ}$ according to the previous study for the optimal trackability threshold of FA [25]. Two regions of interests (ROI) were selected to reconstruct the CST. One ROI was placed on the CST portion of anterior lower pons level, and the other was placed on the CST portion of anterior mid pons level. Fiber tracts passing through both ROIs were designated as the final tract of interest. Patients were subdivided into four groups according to the study classification which was modified from a previous study of DTT in intracerebral hemorrhage [19]. The classification was as following: type A, CSTs are symmetrically preserved; type $\mathrm{B}$, CSTs are preserved, but fiber numbers are asymmetric; type C, CSTs are interrupted at the lesion; and type D, CSTs are not constructed due to degeneration. Patients were categorized again into the DTT-P and as DTT-N group according to the CST integrity in the affected hemisphere. DTT-P group consisted of patients from type A and B groups, and the DTT-N group consisted of patients from type $\mathrm{C}$ and $\mathrm{D}$ groups (Fig. 1). Among the 70 patients which were enrolled in this study, a total 44 patients underwent the DTI tests. The DTI tests and likewise the MEP tests were performed in the most of patients within 5 days after admission to the rehabilitation department. The mean duration time from stroke onset was $31.52 \pm 16.09$ days (range, $5-67$ days). The mean time difference between MEP and DTI tests was $4.73 \pm 7.38$ days (range, 0-34 days). Twenty-six of 44 patients belonged to the DTT-P group, and the other 18 patients belonged to the DTT-N group. The fiber number and FA value of affected hemisphere were obtained in the whole group. The ratio of fiber number and FA value (affected value divided by unaffected value) was calculated in the DTT-P group. While comparing DTT variables during analysis, 11 patients who belonged to type D of our DTT classification were excluded, because such variables were unobtainable due to degeneration of CST.

\section{Clinical evaluation}

Clinical variables including biographical (age, sex), neurological variables (time after onset of stroke, type of lesion, lesion location) and also functional variables were evaluated. Functional variables included the Brunnstrom stage of hand, the upper extremity subscore of Fugl-Meyer assessment (FMAU), the Manual Function Test (MFT),
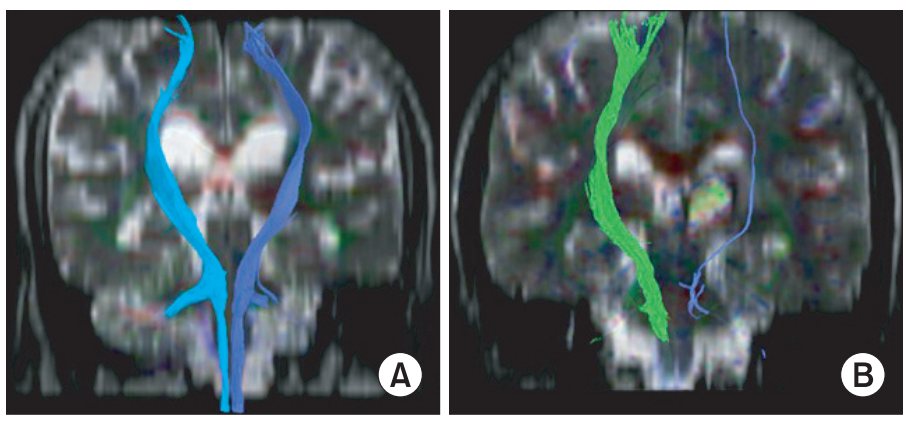

DTT-P
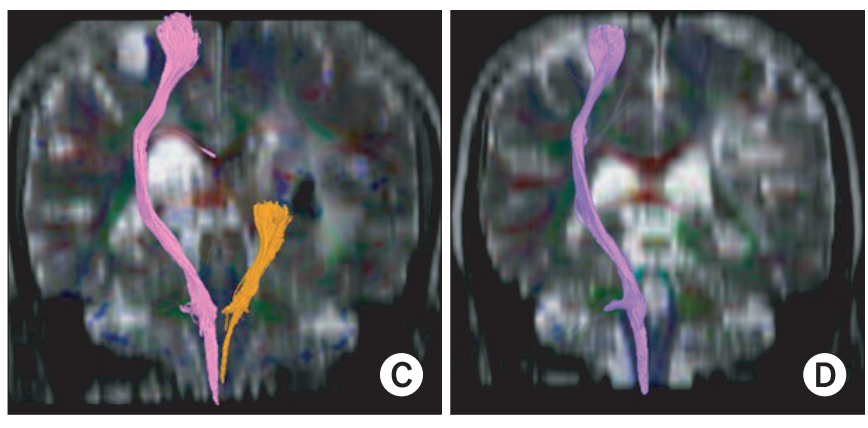

DTT-N

Fig. 1. Classification according to the result of diffusion tensor tractography (DTT). Patients were categorized into DTT-P and DTT-N groups according to integrity of the corticospinal tract (CST) in the affected hemisphere. DTT-P group was consisted of type A and B, and DTT-N group was consisted of type C and D. Type A, CST symmetrically preserved; type B, CST preserved, but asymmetric; type C, CST interrupted at the lesion; and type D, CST not constructed due to degeneration. 
and the Korean version of the Modified Barthel Index (KMBI).

The Fugl-Meyer assessment scale has a 100-point motor, where the point 66 regarded to the upper extremities. This scale is a measure tool for the stroke recovery [26]. The MFT was developed to assess the impairments in motor function of the affected upper extremity of stroke patients. It is composed of 32 items, which examine arm motions and manipulative activities (scores 0-32) [27]. In case of MFT and K-MBI, both admission and discharge scores of patients were obtained, but the admission scores only could be obtained for the Brunnstrom stage and FMAU.

\section{Statistics}

Statistical analysis was performed using SPSS ver. 18.0 software (SPSS Inc, Chicago, IL, USA). The chi-square test and independent t-test were used to analyze differences of biographical and neurological variables, such as lesion type, involved side and time post onset in the MEP-P and MEP-N groups. Depending on the presence or absence of the MEP, functional and DTT variables were compared using the independent t-test. Pearson correlation analysis was used to identify significant correlations between functional variables and MEP parameters. The Fisher exact test was used to show differences of DTT integrity in the MEP-P and MEP-N groups. The Mann-Whitney test and Kruskal-Wallis test were used to prove differences of functional and DTT variables in the combined group of MEP and DTT. Statistical significance was accepted for $\mathrm{p}$ values of $<0.05$.

\section{RESULTS}

Comparison of variables ac cording to MEP responsiveness A total of 70 patients were included in this study, 35 women (50.0\%) and 35 men (50.0\%). Thirty-three patients were in the MEP-P group which showed a presence of MEP response, and 37 patients were in the MEP-N group with an absence of MEP response. Overall mean age was $62.59 \pm 12.38$ years with no significant difference between the two groups. Also the lesion type and the involved side were not significantly different between the two groups. The duration from the onset of stroke to the MEP test was significantly different between the two groups (independent t-test; $25.72 \pm 11.84$ in MEP-P group, 33.35 \pm 16.08 in MEP-N group; $p=0.029$ ) (Table 1), although an effect of the functional variables between both groups was not shown, was proved by analysis of covariance. Binary logistic regression analysis was also performed to estimate the possible biographical and neurological factors that could influence MEP responsiveness, and no significant influence was found.

The Brunnstrom stage and the FMAU in patients on the admission time point to the physical medicine and rehabilitation department were significantly higher in the MEP-P group ( $\mathrm{p}<0.001)$ (Table 2). The admission and discharge scores of MFT and K-MBI were significantly higher in the patients of MEP-P group $(\mathrm{p}<0.001)$. The differences between admission and discharge scores of MFT and K-MBI were obtained by subtracting discharge score by admission score, but the mean scores of differences were not significant $(\triangle \mathrm{MFT}, \mathrm{p}=0.076 ; \Delta \mathrm{K}-\mathrm{MBI}, \mathrm{p}=0.106$; by independent t-test). Among DTT variables, the fiber

Table 1. Biographical and neurological characteristics of subjects

\begin{tabular}{|lccc}
\hline \multicolumn{1}{|c}{ Characteristic } & MEP-P $(\mathbf{n}=\mathbf{3 3})$ & MEP-N $(\mathbf{n}=\mathbf{3 7})$ & p-value \\
\hline Age (yr) & $64.54 \pm 12.08$ & $60.83 \pm 12.54$ & 0.213 \\
\hline Sex (male:female) & $18: 15$ & $17: 20$ & 0.632 \\
\hline Lesion type & & & \\
\hline Infarct:hemorrhage & $21: 12$ & $17: 20$ & 0.157 \\
\hline Cortical:subcortical & $10: 23$ & $5: 32$ & 0.143 \\
\hline Involved side (right:left) & $16: 17$ & $16: 21$ & 0.810 \\
\hline Time post onset (day) & $25.72 \pm 11.84$ & $33.35 \pm 16.08$ & $0.029^{*}$ \\
\hline
\end{tabular}

Values are presented as mean \pm standard deviation or number of subjects.

MEP, motor evoked potential; MEP-P, presence of MEP in affected abductor pollicis brevis; MEP-N, absence of MEP in affected abductor pollicis brevis.

${ }^{*} \mathrm{p}<0.05$ by independent $\mathrm{t}$-test. 
Table 2. Biographical and neurological characteristics of subjects

\begin{tabular}{|c|c|c|c|}
\hline Variable & MEP-P $(\mathbf{n}=33)$ & MEP-N $(n=37)$ & p-value \\
\hline \multicolumn{4}{|l|}{ Brunnstrom } \\
\hline Admission & $4.42 \pm 1.32$ & $1.36 \pm 0.76$ & $<0.001^{*}$ \\
\hline \multicolumn{4}{|l|}{ FMAU } \\
\hline Admission & $47.29 \pm 17.14$ & $6.74 \pm 7.31$ & $<0.001^{*}$ \\
\hline \multicolumn{4}{|l|}{ K-MBI } \\
\hline Admission & $61.45 \pm 23.15$ & $27.35 \pm 23.67$ & $<0.001^{*}$ \\
\hline Discharge & $78.28 \pm 17.91$ & $39.71 \pm 26.03$ & $<0.001^{*}$ \\
\hline$\Delta$ & $16.60 \pm 11.39$ & $12.06 \pm 9.24$ & 0.106 \\
\hline \multicolumn{4}{|l|}{ MFT } \\
\hline Admission & $19.18 \pm 6.81$ & $2.05 \pm 4.48$ & $<0.001^{*}$ \\
\hline Discharge & $22.26 \pm 5.96$ & $4.16 \pm 6.03$ & $<0.001^{*}$ \\
\hline$\Delta$ & $4.09 \pm 3.44$ & $2.29 \pm 3.72$ & 0.076 \\
\hline DTT fiber number (affected) & $719.41 \pm 609.20^{\mathrm{a})}$ & $263.94 \pm 323.95^{\mathrm{b})}$ & $0.012^{*}$ \\
\hline DTT FA value (affected) & $0.53 \pm 0.04^{\mathrm{a})}$ & $0.47 \pm 0.06^{\mathrm{b})}$ & $0.004^{*}$ \\
\hline DTT fiber ratio (affected/unaffected) & $0.69 \pm 0.66^{\mathrm{a})}$ & $0.41 \pm 0.42^{b)}$ & 0.154 \\
\hline DTT FA ratio (affected/unaffected) & $0.98 \pm 0.05^{\text {a) }}$ & $0.88 \pm 0.11^{\mathrm{b})}$ & $0.003^{*}$ \\
\hline
\end{tabular}

Values are presented as mean \pm standard deviation.

MEP, motor evoked potential; MEP-P, presence of MEP in affected abductor pollicis brevis; MEP-N, absence of MEP in affected abductor pollicis brevis; Brunnstrom, Brunnstrom stage of hand; FMAU, upper extremity subscore of FuglMeyer assessment; K-MBI, Korean version of Modified Barthel Index; MFT, Manual Function Test; DTT, diffusion tensor tractography; FA, fractional anisotropy; $\Delta$, difference between admission and discharge scores.

${ }^{\mathrm{a})} \mathrm{n}=17,{ }^{\mathrm{b}} \mathrm{n}=16$; the number of patients who included in MEP-P group and MEP-N group, respectively.

${ }^{*} \mathrm{p}<0.05$ by independent $\mathrm{t}$-test.

Table 3. Correlations between MEP parameters of unaffected hemisphere and clinical variables $(n=70)$

\begin{tabular}{|c|c|c|c|}
\hline \multirow{2}{*}{ Variable } & \multicolumn{3}{|c|}{ Correlation coefficient ( $p$-value) } \\
\hline & RMT & Latency & Amplitude \\
\hline \multicolumn{4}{|l|}{ Brunnstrom } \\
\hline Admission & $-0.044(0.720)$ & $-0.045(0.715)$ & $-0.030(0.805)$ \\
\hline \multicolumn{4}{|l|}{ FMAU } \\
\hline Admission & $-0.029(0.818)$ & $-0.059(0.642)$ & $-0.040(0.753)$ \\
\hline \multicolumn{4}{|l|}{ K-MBI } \\
\hline Admission & $0.074(0.540)$ & $-0.056(0.647)$ & $-0.142(0.241)$ \\
\hline Discharge & $0.005(0.971)$ & $-0.084(0.537)$ & $-0.121(0.376)$ \\
\hline$\Delta$ & $0.076(0.575)$ & $-0.003(0.980)$ & $-0.132(0.334)$ \\
\hline \multicolumn{4}{|l|}{ MFT } \\
\hline Admission & $-0.040(0.742)$ & $-0.042(0.727)$ & $-0.056(0.645)$ \\
\hline Discharge & $-0.054(0.699)$ & $-0.122(0.379)$ & $0.016(0.906)$ \\
\hline$\Delta$ & $-0.015(0.916)$ & $0.065(0.638)$ & $-0.182(0.188)$ \\
\hline
\end{tabular}

MEP, motor evoked potential; RMT, resting motor threshold; Brunnstrom, Brunnstrom stage of hand; FMAU, upper extremity subscore of Fugl-Meyer assessment; K-MBI, Korean version of Modified Barthel Index; MFT, Manual Function Test; $\Delta$, difference between admission and discharge scores.

$\mathrm{p}>0.05$ (Pearson correlation). 
Table 4. Correlations between MEP parameters of unaffected hemisphere and clinical variables $(\mathrm{n}=70)$

\begin{tabular}{|c|c|c|c|}
\hline \multirow{2}{*}{ Variable } & \multicolumn{3}{|c|}{ Correlation coefficient ( $p$-value) } \\
\hline & RMT ratio $^{\text {a) }}$ & Latency ratio $^{\text {a) }}$ & Amplitude ratio ${ }^{a)}$ \\
\hline \multicolumn{4}{|l|}{ Brunnstrom } \\
\hline Admission & $0.123(0.496)$ & $0.079(0.661)$ & $-0.427(0.013)^{*}$ \\
\hline \multicolumn{4}{|l|}{ FMAU } \\
\hline Admission & $0.053(0.776)$ & $0.393(0.029)^{*}$ & $-0.270(0.142)$ \\
\hline \multicolumn{4}{|l|}{ K-MBI } \\
\hline Admission & $0.195(0.276)$ & $0.119(0.509)$ & $-0.380(0.029)^{*}$ \\
\hline Discharge & $0.041(0.844)$ & $0.284(0.169)$ & $-0.063(0.763)$ \\
\hline$\Delta$ & $-0.054(0.798)$ & $0.136(0.516)$ & $0.356(0.081)$ \\
\hline \multicolumn{4}{|l|}{ MFT } \\
\hline Admission & $0.005(0.979)$ & $0.073(0.685)$ & $-0.270(0.129)$ \\
\hline Discharge & $-0.121(0.581)$ & $0.039(0.861)$ & $-0.183(0.404)$ \\
\hline$\Delta$ & $-0.037(0.868)$ & $0.102(0.645)$ & $0.088(0.689)$ \\
\hline Time post onset & $0.253(0.155)$ & $0.030(0.868)$ & $-0.401(0.021)^{*}$ \\
\hline
\end{tabular}

MEP, motor evoked potential; MEP-P, presence of MEP in affected abductor pollicis brevis; RMT, resting motor threshold; Brunnstrom, Brunnstrom stage of hand; FMAU, upper extremity subscore of Fugl-Meyer assessment; K-MBI, Korean version of Modified Barthel Index; MFT, Manual Function Test; $\Delta$, difference between admission and discharge scores.

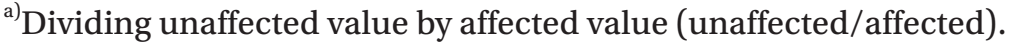

${ }^{*} \mathrm{p}<0.05$ (Pearson correlation).

number, FA value of affected side, and FA ratio (affected value/unaffected value) were significantly different between MEP-P and MEP-N groups, while fiber number ratio was not different (fiber number, $\mathrm{p}=0.012$; FA value, $\mathrm{p}=0.004$; fiber number ratio, $\mathrm{p}=0.154$; FA ratio, $\mathrm{p}=0.003$ ).

\section{Relation of clinical variables and MEP parameters}

The MEP parameters of the unaffected hemisphere, such as RMT, latency, and amplitude, showed no significant correlation with clinical variables in the whole study patient group (Brunnstrom stage, FMAU, MFT, K-MBI; p $>0.05$ by Pearson correlation) (Table 3 ). For 33 subjects in the MEP-P group, correlations between ratio of RMT, latency, amplitude, and clinical variables were analyzed (Table 4). The amplitude ratio, which was calculated by dividing the unaffected value by the affected value, showed a significant negative correlation with the admission scores of Brunnstrom stage $(\mathrm{r}=-0.427, \mathrm{p}=0.013)$ and $\mathrm{K}$-MBI ( $\mathrm{r}=-0.380, \mathrm{p}=0.029)$, while no significant correlation was observed with admission scores of FMAU, MFT and discharge scores of K-MBI, MFT. The amplitude ratio showed a significant negative correlation with time postonset $(\mathrm{r}=-0.401, \mathrm{p}=0.021)$. This may indicate a tendency

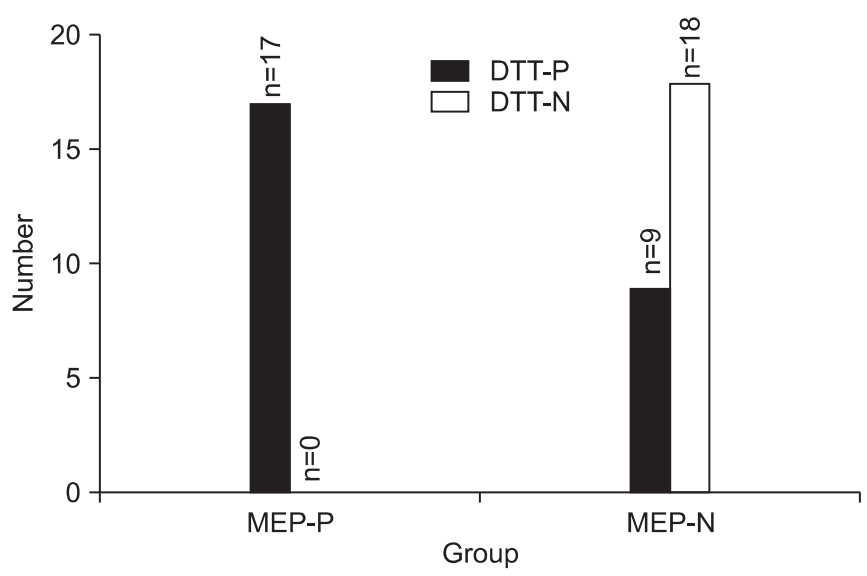

Fig. 2. Classification according to the result of motor evoked potential (MEP) and diffusion tensor tractography (DTT). The distribution of DTT integrity between MEP-P and MEP-N groups were significantly different $(p<0.001$, Fisher exact test).

to return to normal amplitude ratio during the course of time. No significant relation between the RMT ratio and the latency ratio with the clinical variables was shown except the correlation between latency ratio and admission score of FMAU ( $r=0.393, p=0.029)$. 
Table 5. Functional variables according to the presence or absence of MEP and DTT

\begin{tabular}{lcccc}
\multicolumn{1}{c}{ Variable } & MEP-P \& DTT-P $(\mathbf{n}=\mathbf{1 7})$ & Either MEP-P or DTT-P $(\mathbf{n}=\mathbf{9})$ & MEP-N \& DTT-N $(\mathbf{n}=\mathbf{1 8})$ & p-value \\
\hline Brunnstrom & $4.29 \pm 1.45^{\text {a) }}$ & $1.67 \pm 1.32$ & $1.17 \pm 0.38$ & $<0.001^{*}$ \\
FMAU & $43.75 \pm 19.50^{\text {a) }}$ & $11.67 \pm 12.51^{\text {b) }}$ & $4.38 \pm 1.50$ & $<0.001^{*}$ \\
K-MBI & $59.47 \pm 21.99^{\text {a) }}$ & $31.11 \pm 27.04$ & $16.83 \pm 15.24$ & $<0.001^{*}$ \\
MFT & $17.65 \pm 8.05^{\text {a) }}$ & $3.89 \pm 5.18^{\text {b) }}$ & $0.28 \pm 1.18$ & $<0.001^{*}$ \\
\hline
\end{tabular}

Values are presented as mean \pm standard deviation.

MEP, motor evoked potential; MEP-P, presence of MEP in affected abductor pollicis brevis; DTT, diffusion tensor tractography; Brunnstrom, Brunnstrom stage of hand; FMAU, upper extremity subscore of Fugl-Meyer assessment; KMBI, Korean version of Modified Barthel Index; MFT, Manual Function Test.

${ }^{a)} \mathrm{p}<0.05$ by Mann-Whitney test between MEP \& DTT-P and 1 of MEP or DTT-P. ${ }^{\text {b) }} \mathrm{p}<0.05$ by Mann-Whitney test between 1 of MEP or DTT-P and MEP \& DTT-N.

${ }^{*} \mathrm{p}<0.001$ by Kruskal-Wallis test.

\section{Combined study of MEP and DTT}

A total 44 patients who underwent both MEP and DTT were analyzed. All patients $(n=17)$ who were included in MEP-P group belonged to the DTT-P group. Among the 27 patients in MEP-N group, 18 patients (66.7\%) belonged to the DTT-N group, and the other 9 patients (33.3\%) belonged to the DTT-P group. The distribution between groups were significantly distinct $(\mathrm{p}<0.001$ by Fisher exact test) (Fig. 2).

According to the responsiveness of MEP and the integrity of DTT, patients were divided into three groups as both MEP-P and DTT-P group ( $\mathrm{n}=17)$, either MEP-P or DTT-P group ( $\mathrm{n}=9$ ), and both MEP-N and DTT-N group $(\mathrm{n}=18)$ (Table 5). The Brunnstrom stage, FMAU, and MFT on admission showed significant differences between groups, which higher scores were reported in both MEP$P$ and DTT-P group than the other two groups $(p<0.001$ by Kruskal Wallis test).

\section{DISCUSSION}

The attempt of this study was to investigate the factors affecting the MEP responsiveness and parameters and it was also a study goal to find the correlation between the upper extremity function and the combined study of MEP with DTT. According to our results, upper extremity motor function, performance of activities of daily living (ADL) and the time post-onset are the factors which affect the MEP responsiveness and parameters, particularly the amplitude ratio. Also the combination of MEP with DTT is significantly correlated with the upper extremity motor function and the performance of ADL.
It was revealed in many previous studies the presence of MEP response had a prognostic value for motor recovery $[4,8,28]$. As a result of the present study, the Brunnstrom stage, FMAU on admission, and MFT on admission and discharge were significantly higher in the MEP-P group than those in the MEP-N group. The Brunnstrom stage and FMAU are used generally for the assessment of motor recovery after stroke, according to a movement with or without synergy. The MFT is also used in patients after stroke. It tests the motor function of upper extremity from moving in range of motion to manipulating a small object. Therefore, the presence of MEP response is associated with a better motor function of the upper extremity as well as with the motor strength. Moreover, K-MBI scores on patients' admission and discharge were also higher in the MEP-P group. In a previous study K-MBI had been reported to have a higher correlation with MFT [29]. The function of the upper limbs affects the degree of independence in ADL. Accordingly, the presence of a MEP response is also associated with an independent ADL performance. However, the differences between admission and discharge of MFT, K-MBI showed only borderline significances between the MEP-P and MEP-N groups. One possible reason for this insufficient result was the ceiling effect of MFT, K-MBI scores in the MEP-P group, as some of the patients reached almost the maximum scores for both tests. Another reason could be the small sample size for this study, not enough to reach a statistical significance. However, our findings are consistent with a previous study where the correlations between MEP and ADL in stroke patients were analyzed [30]. 
In this study the clinical factors which were correlated with MEP parameters were examined. The MEP amplitude has been known as not suitable for the neurophysiologic status assessment because of the wide variability. So the amplitude ratio can be used as an adequate measurement $[11,12,15]$. In the present study, the amplitude ratio showed a significant correlation with the Brunnstrom stage and the K-MBI score. Although it had only borderline significance, the amplitude ratio was related FMAU and MFT also. Son et al. [15] reported the amplitude ratio reflected the hand strength, but presented the general function of upper extremity and dextrity not well. However, this result suggests that the amplitude ratio reflect the general motor function of upper extremity and ADL performance.

The time post-onset showed a significant correlation with the amplitude ratio. To the best of our knowledge, there has been no study yet about the time influence on the MEP parameters, especially on the amplitude ratio. According to this study, the inter-hemispheric imbalance of amplitude tends to normalize during the course of time, while other MEP parameters, such as RMT and latency, were not significantly affected.

In 44 patients both MEP and DTT were performed, and an analysis between this two tests was conducted. Those 17 patients with a presence of MEP revealed an integrity of the CST on DTT, while 18 (66.7\%) of 27 patients with a MEP absence showed a disruption of the CST on DTT. Both MEP and DTT evaluate the condition of the corticospinal pathway in each physiologic or neurostructural way. However, an absence of MEP could mean nerve cells are dead or have a very high motor threshold [31]. As MEP reflects a relatively long pathway from primary motor cortex to the recording hand muscle through the spinal cord, it might be more vulnerable to degeneration and disruption than DTT. According to the above results, DTT could be considered as a more sensitive tool to evaluate the corticospinal pathway than the MEP test in patients with MEP absence. The study result revealed that the DTT fiber number, FA value of affected side and the FA ratio were significantly higher in the MEP-P group. The DTT fiber number ratio was also higher in the MEP-P group but showed borderline significance only ( $\mathrm{p}=0.154)$, caused probably due to the small sample size. The fiber number is determined by counting the number of voxels contained within a neural tract, and FA value indicates the directional integrity within the white matter [32]. Therefore, a decrease of the fiber number or FA value may estimate the degree of disruption on CST, and it is significantly associated with MEP responsiveness.

In this study, also the clinical variables related with the combination of MEP and DTT were investigated. The previous combined study of MEP and DTT had compared the abilities of MEP and DTT, and investigated the usefulness of the combined study to predict motor outcome $[23,24]$. In the present study, it was found that the group with MEP presence and integrity of CST on DTT showed a significantly higher Brunnstrom stage, FMAU, MFT, and K-MBI scores than the other two groups (either MEP-P or DTT-P group, both MEP-N and DTT-N groups). Through this study result, it may be suggested that the concurrent integrity of the MEP and DTT combination indicate a better upper extremity function and a better ADL also.

This study has a number of limitations. First, the sample size was relative small for the evaluation of various clinical factors. Even so, our analysis yielded many statistically significant findings. Second, those inherent to retrospective studies were also found in our study. Third, a follow up MEP test was not performed to identify the MEP parameter changes in relation to the improvement of clinical variables.

In conclusion, among the MEP parameters, the MEP responsiveness and amplitude ratio are significantly associated with clinical variables which indicate the upper extremity function and the performance of ADL. The inter-hemispheric imbalance of MEP amplitude tends to normalize during the course of time. The combined MEP and DTT tests provide useful informations in indicating the upper extremity function and performance of ADL, and further study involving larger sample size is needed.

\section{CONFLICT OF INTEREST}

No potential conflict of interest relevant to this article was reported.

\section{REFERENCES}

1. Young J, Forster A. Review of stroke rehabilitation. BMJ 2007;334:86-90.

2. Shelton FN, Reding MJ. Effect of lesion location on upper limb motor recovery after stroke. Stroke 
2001;32:107-12.

3. Hendricks HT, Hageman G, van Limbeek J. Prediction of recovery from upper extremity paralysis after stroke by measuring evoked potentials. Scand J Rehabil Med 1997;29:155-9.

4. Escudero JV, Sancho J, Bautista D, Escudero M, LopezTrigo J. Prognostic value of motor evoked potential obtained by transcranial magnetic brain stimulation in motor function recovery in patients with acute ischemic stroke. Stroke 1998;29:1854-9.

5. Jang SH, Kim YH, Chang Y, Han BS, Byun WM, Chang $\mathrm{CH}$. The predictive value of cortical activation by passive movement for motor recovery in stroke patients. Restor Neurol Neurosci 2004;22:59-63.

6. Nelles G, Spiekramann G, Jueptner M, Leonhardt G, Muller S, Gerhard H, et al. Evolution of functional reorganization in hemiplegic stroke: a serial positron emission tomographic activation study. Ann Neurol 1999;46:901-9.

7. Thickbroom GW. Transcranial magnetic stimulation and synaptic plasticity: experimental framework and human models. Exp Brain Res 2007;180:583-93.

8. Pennisi G, Rapisarda G, Bella R, Calabrese V, Maertens De Noordhout A, Delwaide PJ. Absence of response to early transcranial magnetic stimulation in ischemic stroke patients: prognostic value for hand motor recovery. Stroke 1999;30:2666-70.

9. Hendricks HT, van Limbeek J, Geurts AC, Zwarts MJ. Motor recovery after stroke: a systematic review of the literature. Arch Phys Med Rehabil 2002;83:1629-37.

10. Han TR, Bang MS, Lee KW. Motor evoked potentials of upper and lower extremities by magnetic stimulation in hemiparesis. J Korean Acad Rehabil Med 1998;22:386-91.

11. Kamen G. Reliability of motor-evoked potentials during resting and active contraction conditions. Med Sci Sports Exerc 2004;36:1574-9.

12. Christie A, Fling B, Crews RT, Mulwitz LA, Kamen G. Reliability of motor-evoked potentials in the ADM muscle of older adults. J Neurosci Methods 2007;164:320-4.

13. Thickbroom GW, Byrnes ML, Mastaglia FL. A model of the effect of MEP amplitude variation on the accuracy of TMS mapping. Clin Neurophysiol 1999;110:941-3.

14. Maeda F, Keenan JP, Tormos JM, Topka H, PascualLeone A. Interindividual variability of the modulatory effects of repetitive transcranial magnetic stimulation on cortical excitability. Exp Brain Res 2000;133:42530.

15. Son SY, Park SH, Seo JH, Ko MH. Correlation of the motor evoked potentials amplitude and hand function of the affected side in stroke. J Korean Acad Rehabil Med 2011;35:34-41.

16. Basser PJ, Pierpaoli C. Microstructural and physiological features of tissues elucidated by quantitativediffusion-tensor MRI. J Magn Reson B 1996;111:20919.

17. Mori S, Crain BJ, Chacko VP, van Zijl PC. Threedimensional tracking of axonal projections in the brain by magnetic resonance imaging. Ann Neurol 1999;45:265-9.

18. Puig J, Pedraza S, Blasco G, Daunis-I-Estadella J, Prats A, Prados F, et al. Wallerian degeneration in the corticospinal tract evaluated by diffusion tensor imaging correlates with motor deficit 30 days after middle cerebral artery ischemic stroke. AJNR Am J Neuroradiol 2010;31:1324-30.

19. Cho SH, Kim SH, Choi BY, Cho SH, Kang JH, Lee CH, et al. Motor outcome according to diffusion tensor tractography findings in the early stage of intracerebral hemorrhage. Neurosci Lett 2007;421:142-6.

20. Yoshioka H, Horikoshi T, Aoki S, Hori M, Ishigame $\mathrm{K}$, Uchida $\mathrm{M}$, et al. Diffusion tensor tractography predicts motor functional outcome in patients with spontaneous intracerebral hemorrhage. Neurosurgery 2008;62:97-103.

21. Jang SH. Prediction of motor outcome for hemiparetic stroke patients using diffusion tensor imaging: a review. NeuroRehabilitation 2010;27:367-72.

22. Cho SH, Kim DG, Kim DS, Kim YH, Lee CH, Jang SH. Motor outcome according to the integrity of the corticospinal tract determined by diffusion tensor tractography in the early stage of corona radiata infarct. Neurosci Lett 2007;426:123-7.

23. Kwon YH, Son SM, Lee J, Bai DS, Jang SH. Combined study of transcranial magnetic stimulation and diffusion tensor tractography for prediction of motor outcome in patients with corona radiata infarct. J Rehabil Med 2011;43:430-4.

24. Jang SH, Ahn SH, Sakong J, Byun WM, Choi BY, Chang $\mathrm{CH}$, et al. Comparison of TMS and DTT for predicting motor outcome in intracerebral hemorrhage. J Neurol 
Sci 2010;290:107-11.

25. Kunimatsu A, Aoki S, Masutani Y, Abe O, Hayashi N, Mori $\mathrm{H}$, et al. The optimal trackability threshold of fractional anisotropy for diffusion tensor tractography of the corticospinal tract. Magn Reson Med Sci 2004;3:11-7.

26. Gladstone DJ, Danells CJ, Black SE. The Fugl-Meyer assessment of motor recovery after stroke: a critical review of its measurement properties. Neurorehabil Neural Repair 2002;16:232-40.

27. Nakamura R. Manual function test (MFT) and functional occupational therapy for stroke patients. Tokorozawa, Japan: National Rehabilitation Center for the Disabled; 2000.

28. Timmerhuis TP, Hageman G, Oosterloo SJ, Rozeboom AR. The prognostic value of cortical magnetic stimulation in acute middle cerebral artery infarction compared to other parameters. Clin Neurol Neurosurg
1996;98:231-6.

29. Miyamoto S, Kondo T, Suzukamo Y, Michimata A, Izumi S. Reliability and validity of the manual function test in patients with stroke. Am J Phys Med Rehabil 2009;88:247-55.

30. Lim KB, Kim JA. Activity of daily living and motor evoked potentials in the subacute stroke patients. Ann Rehabil Med 2013;37:82-7.

31. Rossini PM, Barker AT, Berardelli A, Caramia MD, Caruso G, Cracco RQ, et al. Non-invasive electrical and magnetic stimulation of the brain, spinal cord and roots: basic principles and procedures for routine clinical application. Report of an IFCN committee. Electroencephalogr Clin Neurophysiol 1994;91:79-92.

32. Alexander AL, Lee JE, Lazar M, Field AS. Diffusion tensor imaging of the brain. Neurotherapeutics 2007;4:316-29. 